\section{Chronic Respiratory}

Failure in

Limb-Girdle

Muscular Dystrophy: Successful Long-Term Therapy With Nasal Bilevel Positive

\section{Airway Pressure}

\author{
Patricia L. Robertson, MD ${ }^{* \dagger}$ and Dietrich \\ W. Roloff, MD*
}

Chronic respiratory failure is a major factor contributing to mortality in progressive neuromuscular disorders. Among the muscular dystrophies, respiratory failure most commonly occurs with Duchenne dystrophy, while in Becker, limb-girdle, and facioscapulohumeral dystrophies, respiratory failure is infrequent and generally occurs in the more severe cases that have progressed to a nonambulatory, advanced functional stage. We report two brothers with a myopathic disease in which the distribution of weakness, initial clinical course, heredity, and muscle pathology most closely resembled a limb-girdle type of dystrophy. Both brothers, however, presented with chronic alveolar hypoventilation and respiratory failure when their locomotor disabilities were still mild. Respiratory failure was reversed, and satisfactory ventilation has been maintained for more than a year using a type of noninvasive intermittent positive pressure ventilation, with a bilevel positive airway pressure device (BiPAP), administered through a nasal mask during sleeping hours. These cases demonstrate an unusual presentation of limb-girdle dystrophy, and document that nocturnal, nasal administration of continuous airway pressure using the Bi-PAP device may be sufficient to maintain adequate long-term ventilation in some patients with neuromuscular causes of respirato- ry failure, and thus significantly improve quality of life and delay the need for more complex or invasive forms of assisted ventilation.

Robertson PL, Roloff DW. Chronic respiratory failure in limb-girdle muscular dystrophy: Successful long-term therapy with nasal bilevel positive airway pressure. Pediatr Neurol 1994;10:328-331.

\section{Introduction}

Chronic respiratory failure contributes to mortality in progressive neuromuscular disorders, usually after they have progressed to severe locomotor dysfunction when ambulation is lost. This failure is most commonly seen in Duchenne dystrophy, but sometimes occurs in Becker, limb-girdle, and facioscapulohumeral dystrophies, in which respiratory failure also parallels more severe locomotor incapacity $[1,2]$. We present two brothers with a myopathic disease in which the distribution of weakness, clinical course, heredity, and muscle pathology most closely resembled a limb-girdle type of dystrophy. Both brothers presented with chronic alveolar hypoventilation and respiratory failure when their motor functional disabilities were still mild. We report their response to the nocturnal application of noninvasive intermittent positive pressure ventilation (NIPPV) with a bilevel positive airway pressure device (Bi-PAP), which delivers different levels of inspiratory positive airway pressure (IPAP) and expiratory positive airway pressure (EPAP) through a nasal mask.

\section{Methods}

The patients were treated with a form of nocturnal NIPPV employing a device for delivering bilevel positive airway pressure (Bi-PAP Respironics Inc., Monroeville, PA), consisting of an appropriately-sized mask attached over the nose with a chin strap, through which a blower and solenoid valves supply and regulate inspiratory and expiratory pressures. When the patient initiates a breath, a positive pressure gradient results, delivering a small assist to inspiration. During the rest of the ventilatory cycle, a lower level of positive pressure is supplied which prevents obstruction of the airway.

\section{Case Reports}

Case 1. A 14-year-old boy presented to the emergency room with complaints of fatigue, shortness of breath, orthopnea, and increasing daytime somnolence. Past medical history was remarkable for gradually increasing muscle weakness over the preceding 2 years, greater in the legs than arms, with increasing difficulty climbing stairs. Early motor milestones had been normal in infancy and early childhood. A muscle biopsy performed 6 months earlier indicated end-stage muscle with substantial fat and connective tissue replacement between islands of muscle
From the Departments of *Pediatrics and ${ }^{\dagger}$ Neurology; The University of Michigan; Ann Arbor, Michigan.
Communications should be addressed to:

Dr. Robertson; Division of Pediatric Neurology; University of Michigan Medical School; L2220 Women's Building; Ann Arbor, MI 48109-0570.

Received December 16, 1993; accepted April 5, 1994. 
fibers, a few degenerating muscle fibers, and a larger number of split or angular atrophic fibers and fibers with pyknotic nuclei. There was no fiber-type grouping and cytochemistry for acid maltase was normal. The patient had a 19-year-old brother who was not very athletic but was considered normal by the family, as were all other family members. The parents were first cousins of Arabic descent, with other consanguineous marriages in the kindred.

On examination, the boy's weight was at the 80 th percentile and height at the 5 th percentile; heart rate was $120 / \mathrm{min}$ with a normal $S_{1}$ and $S_{2}$, an $\mathrm{S}_{3}$ gallop, and no murmur; respiratory rate was $28 / \mathrm{min}$; rhonchi were heard in the upper lung fields with slightly decreased breath sounds at the bases. He was somnolent, but had no other abnormalities of mental status. He had mild bilateral facial weakness and slightly hyponasal speech. His motor strength was $4-$ to $4 / 5$ in proximal upper and lower extremity girdle muscles, and $4+$ to $5 / 5$ in distal upper and lower extremity muscles; he used a Gowers' maneuver to rise from the floor Sensation was intact.

Initial respiratory function parameters were: tidal volume, $0.12 \mathrm{~L}$ ( $15 \%$ predicted); vital capacity, $0.25 \mathrm{~L}$ ( $8 \%$ predicted); negative inspiratory force, $-18 \mathrm{~cm} \mathrm{H}_{2} \mathrm{O}$ (45\% predicted); oxygen saturation on room air, $86 \%$. When he was placed on $100 \%$ oxygen, his arterial blood gases were: $\mathrm{pH}, 7.27 ; \mathrm{PaCO}_{2}, 70$ torr; $\mathrm{PaO}_{2}, 181$ torr; and $\mathrm{HCO}_{3}, 33 \mathrm{mEg} / \mathrm{L}$. Some basilar atelectasis, initially present on chest $\mathrm{x}$-ray, disappeared after assisted ventilation was begun. On electrocardiography, there was mild right axis deviation and right atrial enlargement. On electromyography, there was no abnormal insertional or spontaneous activity; there were low amplitude, short duration motor unit potentials with a full recruitment pattern. Nerve condition velocities were normal. Serum creatine kinase was $20 \mathrm{IU} / \mathrm{L}$.

He was intubated immediately and received mechanical positive pressure ventilation for 2 weeks. After extubation, a polysomnogram was recorded in the supine and lateral decubitus position during 6 hours of sleep. There were 156 periods of hypopnea which were not obstructive, averaged $45 \mathrm{~s}$, and extended to $94 \mathrm{~s}$ in duration; 61 obstructive apneas occurred during this time. Baseline oxygen saturation fell to $80 \%$ during sleep, with desaturation to as low as $57 \%$ during the hypopneas, which were longer in REM sleep and in the supine position. An excessive number of arousals from sleep occurred during the period of recording. Nocturnal NIPPV using the Bi-PAP device was then begun; at IPAP and EPAP settings adjusted to 13 and $7 \mathrm{~cm} \mathrm{H}_{2} \mathrm{O}$, along with $1.5 \mathrm{~L}$ oxygen, the oxygen desaturations disappeared, and the obstructive events and nonobstructive periods of hypopnea decreased by $80-85 \%$. The improvement was much less complete when positive airway pressure was maintained at the same level during inspiration and expiration. Nocturnal therapy has continued for more than 1 year without respiratory or somnolence symptoms; he attends school regularly and has experienced no further deterioration of locomotor function.

Case 2. The 19-year-old brother of Patient 1 was admitted with a 1 month history of nocturnal dyspnea and a 2-day history of dyspnea during the day. He had been a poor athlete, and during the preceding year had experienced more difficulty climbing stairs, which he attributed to a slight weight gain. Several months prior to admission, while visiting his hospitalized brother, he was observed to have mild proximal weakness. Both brothers were then presumed to have the same disease, and he underwent a muscle biopsy which revealed marked variation in fiber size with pronounced fiber splitting and many internalized nuclei. An increased amount of fat and connective tissue with replacement of normal fibers was also seen. Atrophic fibers were of both type I and type II, there was no fiber type grouping, and cytochemistry for lipid, glycogen, phosphorylase, phosphatases, acid maltase, and esterase were normal. No abnormalities of mitochondria were evident by electron microscopic examination, and biochemical analysis of mitochondrial enzymes was normal. The biopsy was thought to be consistent with a dystrophic process and most suggestive of a facioscapulohumeral or limb-girdle type of dystrophy. Dystrophin analysis of muscle was normal.

On examination, his weight was at the 75 th percentile and his height at the 10 th percentile. His respiratory rate was $36 / \mathrm{min}$; he had a normal cardiac examination and minimal bilateral basilar rales on chest auscul- tation. His mental status was normal; he had very mild impairment of eye closure and buccal muscle strength. Muscle tone and bulk were normal; strength was 4 to $4+15$ in proximal upper and lower extremity girdle muscles, and $5-$ to $5 / 5$ in distal upper and lower extremity muscles. He performed a partial Gowers' maneuver to rise from the floor. Sensation was normal.

On laboratory examination, his arterial blood gases on room air were: $\mathrm{pH}, 7.42 ; \mathrm{PaCO}_{2}, 50$ torr; $\mathrm{PaO}_{2}, 61$ torr; $\mathrm{HCO}_{3}, 33 \mathrm{mEq} / \mathrm{L}$, with an oxygen saturation of $91 \%$. Pulmonary function parameters were: tidal volume, $0.25 \mathrm{~L}$ ( $30 \%$ predicted); vital capacity, $1.3 \mathrm{~L}$ (33\% predicted); and negative inspiratory force, $-40 \mathrm{~cm} \mathrm{H}_{2} \mathrm{O}$ (100\% predicted). Electrocardiography was normal. Electromyography revealed short duration motor unit potentials with low amplitude and normal recruitment sug. gestive of a myopathic process. During 6 hours of sleep recorded on a polysomnogram, there were 42 obstructive apneas and 208 periods of hypopnea; approximately half of the hypopneas were obstructive and their average duration was $24 \mathrm{~s}$; they were longer and associated with greater oxygen desaturation during REM sleep; sleep position was left lateral decubitus for the entire period. Baseline oxygen saturation in sleep was $81 \%$, and worsened to $30 \%$ during episodes of obstructive apnea or hypopnea. There were also an increased number of arousals from sleep and abnormal sleep architecture.

NIPPV using a nasal mask and Bi-PAP device was instituted during sleep. At IPAP and EPAP settings of 13 and $7 \mathrm{~cm} \mathrm{H}_{2} \mathrm{O}$, respectively, with $1 \mathrm{~L}$ oxygen, the hypoventilation, obstructive events, and desaturation during them decreased maximally. At these settings, oxygen desaturations disappeared and obstructive events and nonobstructive periods of hypopnea decreased by $85-90 \%$. Trials with continuous airway pressure at the same level during inspiration and expiration, at several different pressures, did not produce as much improvement. The patient has had continued relief of respiratory symptoms on this therapy for more than 1 year, during which time he has been able to work and attend community college.

\section{Discussion}

The patients in these 2 cases initially presented a somewhat confusing diagnostic problem. The initial clinical examination of the younger brother, along with electromyography, suggested a myopathic process. The histologic appearance of his muscle was confirmatory, but the muscle disease was advanced to a degree that a more specific diagnosis could not be made. A congenital myopathy was a diagnostic possibility in view of the respiratory failure along with a relatively mild degree of extremity weakness, a finding that can occur in some of these myopathies [3,4]. Myotonic dystrophy is sometimes also associated with early respiratory disturbances, but he lacked other clinical features and the electrical phenomena of myotonia [5].

The biopsy obtained from the less severely involved older brother was more informative, showing features most consistent with a dystrophic muscular process. These findings excluded a congenital myopathy. The clinical presentation as well as the normal dystrophin analysis excluded both Duchenne and Becker form of X-linked dystrophy. Taken together, the histologic appearance of muscle, the clinical pattern of proximal extremity weakness with only mild facial involvement, and the history of consanguinity most strongly suggested an autosomal recessive type of limb-girdle dystrophy. The presentation with early respiratory failure was the feature difficult to reconcile with this diagnosis. 
Chronic respiratory failure has been reported in association with limb-girdle dystrophy, but in only a few cases. Sixty patients with well established limb-girdle dystrophy of 11-15 years duration had minimal changes in respiratory function measured spirometrically, and no evidence of respiratory hypoventilation, even in those who were not ambulatory [6]. Mild pulmonary function abnormalities were found in several other patients with limb-girdle dystrophy, but significant abnormality of ventilation was evident on blood gas determination in only 3 patients who were already wheelchair-bound $[7,8]$. Two additional patients with limb-girdle dystrophy whose motor functional status was not reported had spirometric pulmonary function abnormalities, $\mathrm{CO}_{2}$ retention, and weakness of diaphragm muscles measured by transdiaphragmatic pressure change [9]. The diaphragm weakness was thought to be the most likely reason for their hypoventilation because the degree of diaphragm weakness correlated well with the degree of $\mathrm{CO}_{2}$ retention, particularly at night, when it was greater than during the day. A muscular dystrophy with a limb-girdle distribution of weakness and an autosomal recessive form of inheritance was described in 49 children from 17 Arabic families in Tunisia. However, these patients had earlier onset of symptoms and a more rapidly progressive course to loss of ambulation without respiratory failure, making it less like the disease in our patients [10].

Thus, although not common, respiratory and especially diaphragm muscle weakness can occur in limb-girdle dystrophy, and seems likely to be the primary etiology of the respiratory failure in our patients. Patients with limbgirdle dystrophy presenting with such early, severe respiratory muscle weakness while they are still ambulatory have not been previously reported.

Adequate ventilation has been maintained for more than 1 year in each of our patients with limb-girdle dystrophy and respiratory failure, solely by the administration of NIPPV using the nasally applied Bi-PAP device. This system is more convenient and less invasive than either negative pressure or tracheostomy-delivered ventilation, which have also been used to support respiratory function in patients with various neuromuscular disorders [11-13]. It is better tolerated than mouth-administered volume ventilation $[12,14]$ and is less expensive than nasally administered volume ventilators, which are more elaborate than necessary for patients who require only this degree of nocturnal support [15]. It also affords the patient primary control of respiratory frequency, inspiratory flow, and expiratory time, making it more comfortable and better tolerated than volume ventilation.

The Bi-PAP system may prevent airway obstruction in the same way that single level continuous positive airway pressure (CPAP) does, by splinting open an occluded airway during expiration [16]. This mechanism accounts for the disappearance in both brothers of the obstructive apnea likely due to pharyngeal muscle weakness. But other mechanisms must contribute to the improvement of the nonobstructive chronic hypopnea that also occurred in these patients. Bi-PAP seems to provide a boost to inspiratory effort that is greater than could be tolerated if the maximum pressure was continued into expiration. Indeed, when the pressures were adjusted during polysomnographic recording to determine the optimal levels for these patients, there was less improvement in the nonobstructive component of the hypoventilation when CPAP was used instead of Bi-PAP. Bi-PAP has also been shown to reduce respiratory muscle effort, which, by providing rest for fatigued muscles during the night, may be the basis for the sustained ventilatory improvement that persisted into daytime hours in our patients $[17,18]$. The improvement of the chronic hypopnea also should delay the occurrence of pulmonary hypertension that would develop secondary to chronic $\mathrm{CO}_{2}$ retention.

With their early onset of respiratory failure, the patients described in this report demonstrate an unusual course of limb-girdle type of muscular dystrophy. Moreover, they illustrate that respiratory failure should be recognized as a possible cause of progressive fatigue in patients with this or other undiagnosed neuromuscular problems.

This form of nocturnal intermittent positive pressure ventilation employing the Bi-PAP device with a nasal mask has been well tolerated in these teenagers and has effectively assisted their respiratory impairment for more than a year; it significantly improved their symptoms and quality of life without resorting to more invasive or restrictive forms of support. This noninvasive method of assisting ventilation may be effective in other patients with chronic respiratory failure of neuromuscular etiologies as well, and could delay the need for more invasive and inconvenient forms of therapy in a number of them. Moreover, this method could prolong meaningful survival and improve the quality of life of patients for whom more invasive methods of mechanical ventilation may not be considered.

\section{References}

[1] Inkley SR, Oldenburg FC, Vignos PJ. Pulmonary function in Duchenne muscular dystrophy related to stage of disease. Am J Med 1974;56:297-306.

[2] Fowler WM, Nayak NN. Slowly progressive proximal weakness: limb girdle syndrome. Arch Phys Med Rehab 1983;64:527-37.

[3] Heckmatt JZ. Respiratory care in muscular dystrophy. Br Med J 1987;295:1014-15.

[4] Maayan C, Springer C, Armon Y, Bar-Yishay E, Shapira Y, Godfrey $\mathrm{S}$. Nemaline myopathy as a cause of sleep hypoventilation. Pediatrics 1986;77:390-5.

[5] Benaim S, Worster-Drought C. Myotonic dystrophy with myotonia of the diaphragm causing pulmonary hypoventilation and anoxaemia and secondary polycythemia. Med Illust 1956;8:221-6.

[6] Rideau Y, Jankowski LW, Grellet J. Respiratory function in the muscular dystrophies. Muscle Nerve 1981;4:155-64.

[7] Neustadt JE, Levy RC, Speigel IJ, Carbon dioxide narcosis in association with muscular dystrophy. JAMA 1964;187:616-7.

[8] Contamin F, Lissac J, Nick J. Late myopathy with alveolar hypoventilation caused by complete paralysis of the diaphragm. Diagnostic and therapeutic problems. Presse Med 1966;74:1939-42. 
[9] Newsom Davis J, Loh L. Alveolar hypoventilation and respiratory muscle weakness. Bull Eur Physiopath Resp 1979;15:45-51.

[10] Ben Hamida M, Fardeau M. Severe, autosomal recessive limbgirdle dystrophies frequent in Tunisia. In: Angelini C, Danieli GA, Fontanari D, eds. Muscular dystrophy research. Advances and new trends. Amsterdam: Excerpta Medica, 1980.

[11] Braun SR, Sufit RL, Giovannoni R, O'Connor M, Peters H. Intermittent negative pressure ventilation in the treatment of respiratory failure in progressive neuromuscular disease. Neurology 1987;37:1874-5.

[12] Bach JR, Alba A, Mosher R, Delaubier A: Intermittent positive pressure ventilation via nasal access in the management of respiratory insufficiency. Chest 1987;92:168-70.

[13] Heckmatt JZ, Loh L, Dubowitz V. Nocturnal hypoventilation in children with nonprogressive neuromuscular disease. Pediatrics 1989; 83:250-5.

[14] Bach JR, Alba AS, Bohatiuk G, Saporito L, Lee M. Mouth intermittent positive pressure ventilation in the management of postpolio respiratory insufficiency. Chest 1987;91:859-64.

[15] Waldhorn RE: Nocturnal nasal intermittent positive pressure ventilation with bi-level positive airway pressure (Bi-PAP) in respiratory failure. Chest 1992;101:516-21.

[16] Strohl KP, Redline S. Nasal CPAP therapy, upper airway muscle activation, and obstructive sleep apnea. Am Rev Resp Dis 1986;134: 555-8.

[17] Petraf BJ, Kimoff RJ, Levy RD, Cosio MG, Gottfried SB. Nasal continuous positive airway pressure facilitates respiratory muscle function during sleep in severe chronic obstructive pulmonary disease. Am Rev Resp Dis 1988;143:928-35.

[18] Alexander DJ, Wakai Y, Road J, Wilcox PG. Bi-level positive airway pressure (Bi-PAP) for ventilatory muscle support in patients with chest wall and neuromuscular disorders [abstract]. Am Rev Resp Dis 1991;143:4 